\title{
Variabilidade espacial e correlação dos atributos do solo com produtividade do milho e da soja
}

\author{
Evandro GELAIN ${ }^{1}$, Eduardo Leonel BOTTEGA2*, \\ Anamari Viegas de Araujo MOTOMIYA ${ }^{1}$, Zanandra Boff de OLIVEIRA ${ }^{2}$ \\ ${ }^{1}$ Universidade Federal da Grande Dourados, Dourados, MS, Brasil. \\ ${ }^{2}$ Universidade Federal de Santa Maria, Santa Maria, RS, Brasil. \\ *E-mail: eduardo.bottega@ufsm.br \\ (ORCID: 0000-0003-0585-5318; 0000-0003-4035-6880; 0000-0003-2170-8676; 0000-0003-3422-8452)
}

Recebido em 21/01/2021; Aceito em 29/11/2021; Publicado em 17/12/2021.

\begin{abstract}
RESUMO: O emprego de técnicas de agricultura de precisão, associadas a análises geoestatísticas, possibilita mapear a variabilidade espacial existente em um campo de produção. O conhecimento da variabilidade é importante ferramenta na tomada de decisões quanto ao manejo da área, uma vez que possibilita que este seja realizado de forma localizada. O estudo foi realizado em um talhão da Fazenda Planalto, localizada no município de Maracaju - MS, com o objetivo de avaliar a variabilidade espacial e correlação entre os atributos químicos e granulométricos do solo e a produtividade do milho e da soja. Foi utilizada uma grade amostral contendo 187 pontos, utilizando-se 10 amostras simples de solo por ponto amostral. Não foi detectado dependência espacial para o cálcio, alumínio, acidez potencial, soma de bases, ferro e zinco. As melhores estimativas em locais não amostrados foram obtidos para a areia e argila. A produtividade da soja apresentou forte dependência espacial e se correlacionou positivamente de forma forte com o fósforo e moderada com o magnésio.
\end{abstract}

Palavras-chave: Latossolo; dependência espacial; Glycine max; Zea mays.

\section{Spatial variability and correlation of chemicals and physical soil attributes with corn and soybean yield}

\begin{abstract}
The use of precision farming techniques, associated with geostatistical analysis, makes it possible to map the spatial variability in a production field. The knowledge of variability is an important tool in decision making regarding the management of the area, since it allows it to be carried out in a localized manner. The study was carried out in a plot of Fazenda Planalto, located in the municipality of Maracaju - MS, with the objective of evaluating the spatial variability and correlation between the chemical and granulometric attributes of the soil and the corn and soybeans yield. A sampling grid containing 187 points was used, using 10 simple soil samples per sample point. No spatial dependence was detected for calcium, aluminum, potential acidity, sum of bases, iron and zinc. The best estimates in unsampled locations were obtained for sand and clay. The soybeans yield showed strong spatial dependence and was positively correlated strongly with phosphorus and moderately with magnesium.

Keywords: Oxisol; spatial dependence; Glycine max; Zea mays.
\end{abstract}

\section{INTRODUÇÃO}

$O$ advento da mecanização agrícola foi um dos responsáveis pelo aumento da produção agrícola mundial, isso ocorreu em função de mudanças no processo de produção, destacando-se a possibilidade de cultivo em extensas áreas. $\mathrm{Na}$ agricultura moderna, a utilização dos insumos agrícolas é empregada de forma a otimizar sua eficiência, ou seja, utilizar a quantidade adequada, no momento correto. Isto contribui para a redução da contaminação do ambiente e aumento na margem de lucro do agricultor.

Neste cenário, a agricultura de precisão vem ganhando espaço como importante ferramenta do sistema produtivo atual. De acordo com Baerdemaeker (2013) os princípios básicos da agricultura de precisão podem ser vistos como um resumo das boas práticas agrícolas que requerem a correta coleta, análise e utilização de informações sobre o solo, histórico da produtividade da área, o correto emprego de produtos químicos/biológicos (no momento e local correto utilizando dos equipamentos corretos nas aplicações).

Neste contexto, o conhecimento da variabilidade espacial e temporal, em especial dos atributos do solo, é destacado. O conhecimento da variabilidade espacial dos atributos químicos e físicos do solo é destacado em diversos estudos, nos quais é relatado que o conhecimento desta variação ao longo do tempo e espaço se faz necessário para orientar estratégias de manejo agrícola (CRUZ et al., 2011; OLIVEIRA et al., 2013; CERRI; MAGALHÃES, 2012).

Estudos recentes têm demonstrado que o solo está naturalmente sujeito à variação de suas propriedades físicas e químicas no espaço e no tempo, resultante da complexa interação entre pedologia, topografia e clima (ARAÚJO et al., 2018). A modelagem desta variabilidade é possível utilizando 
de técnicas geoestatísticas. A geoestatística permite realizar o estudo da variabilidade espacial dos atributos do solo através de modelos teóricos de variogramas, efetuando a estimativa de valores em locais não amostrados por meio da krigagem, o que possibilita a confecção de mapas temáticos dos atributos de interesse na área estudada (SILVA et al., 2010).

Como já ressaltado, conhecer a variabilidade espacial dos atributos do solo é de fundamental importância para tomada de decisão quanto ao manejo a ser adotado. Além disso, tal informação pode explicar variações observadas na produtividade das culturas, uma vez que os mapas de produtividade ilustram esta variabilidade (GUO et al., 2012).

Diante do exposto, o objetivo deste trabalho foi avaliar a variabilidade espacial e a correlação dos atributos do solo, da produtividade de milho e da produtividade de soja, em uma área comercial de produção agrícola, cultivada sob semeadura direta no sistema de sucessão de culturas: soja / milho safrinha + braquiária (Urochloa ruziziensis).

\section{MATERIAL E MÉTODOS}

O presente estudo foi conduzido na Fazenda Planalto, no município de Maracajú - MS. A propriedade apresenta coordenadas geográficas de $21^{\circ} 38^{\prime} 20^{\prime \prime}$ de latitude Sul e $55^{\circ}$ 33' 30" de longitude Oeste. A altitude média em relação ao nível do mar é de $550 \mathrm{~m}$. De acordo com a classificação de Köppen, o clima da região é classificado como Am (Alvares et al., 2013), com verão chuvoso e inverno seco, com temperatura média acima de $18^{\circ} \mathrm{C}$ no mês mais frio. Apresenta ainda precipitação média anual de 1500 a 1700 $\mathrm{mm}$.

A coleta de dados foi realizada em um talhão com área de 101 ha. O solo área é classificado como Latossolo Vermelho Distroférrico, de textura argilosa a muito argilosa (EMBRAPA, 2013). A área vem sendo cultivada, nos últimos 20 anos, sob semeadura direta.

$\mathrm{Na}$ semeadura da cultura da soja utilizou-se $150 \mathrm{~kg} \mathrm{ha}^{-1}$ de MAP, depositados na linha. Para a implantação da cultura do milho safrinha, aplicou-se $100 \mathrm{~kg} \mathrm{ha}^{-1}$ de Ureia na linha e $200 \mathrm{~kg} \mathrm{ha}^{-1}$ de $\mathrm{KCl}$ em cobertura. Nas safras de 2005/06 e 2006/07 o talhão foi utilizado com pastagem de capim tanzânia (Panicum maximum cv. Tanzânia) não sendo realizada adubação. Nas safras de 2007/08 a 2012/13, a área foi cultivada sob sucessão de soja/milho safrinha + braquiária, com adubação de $150 \mathrm{~kg} \mathrm{ha}^{-1}$ de MAP no sulco de semeadura da soja e de $100 \mathrm{~kg} \mathrm{ha}^{-1}$ de Uréia no sulco de semeadura do milho safrinha + braquiária, complementando com $200 \mathrm{~kg}$ $\mathrm{ha}^{-1}$ de $\mathrm{KCl}$ em cobertura.

$\mathrm{Na}$ coleta de solo, para o mapeamento da variabilidade espacial dos atributos químicos e granulométricos, foi utilizada uma grade amostral composta por 187 pontos georreferenciados (Figura 1). Para a navegação aos pontos amostrais utilizou-se um aparelho receptor GPS Garmin, Modelo HCx.

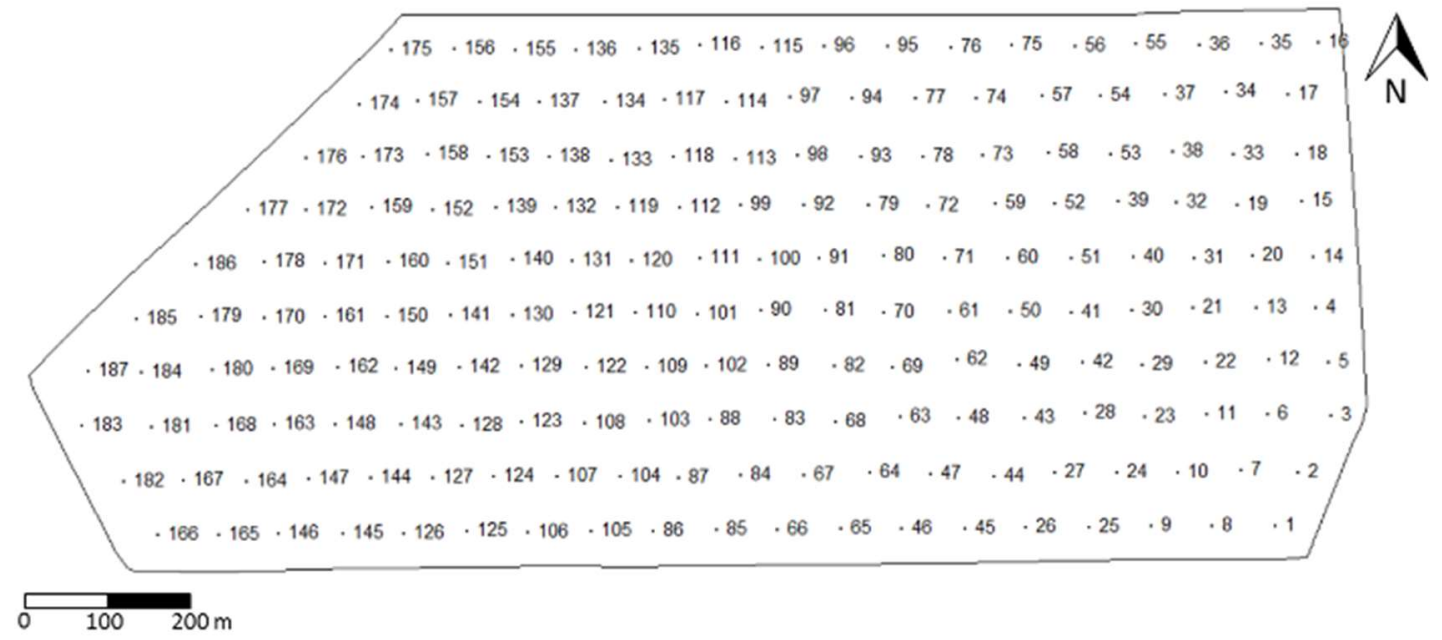

Figura 1. Grade amostral utilizada para coleta de solo.

Figure 1. Sampling grid used for date collection.

Em um raio de aproximadamente 10 metros de cada ponto amostral, foram retiradas 10 amostras simples de solo, representativas da profundidade de $0,00-0,20 \mathrm{~m}$. Utilizouse, para coleta do solo, uma broca helicoidal de uma polegada de diâmetro acoplada a uma furadeira.

Foram avaliados os seguintes atributos químicos do solo: $\mathrm{pH}$ em $\mathrm{CaCl}_{2}\left(0,01 \mathrm{~mol} \mathrm{~L}^{-1}\right)$, potássio $\left(\mathrm{K}^{+}\right)$, cálcio $\left(\mathrm{Ca}^{+2}\right)$, magnésio $\left(\mathrm{Mg}^{+2}\right)$ e hidrogênio mais alumínio $\left(\mathrm{H}^{+}+\mathrm{Al}^{+3}\right)$ trocáveis determinados de acordo com Claessen (1997). A soma de bases (SB) foi estimada pelo somatório das bases trocáveis no solo. Utilizando a equação $\mathrm{T}=\mathrm{SB}+\left(\mathrm{H}^{+}+\right.$ $\mathrm{Al}^{+3}$ ), estimou-se a capacidade de troca de cátions do solo (T). A saturação por bases (V) foi estimada pela expressão: $V$ $=100 \times \mathrm{SB} / \mathrm{T}$.

A granulometria do solo foi determinada por meio da dispersão do solo em solução com $\mathrm{NaOH}\left(0,1 \mathrm{~mol} \mathrm{~L}^{-1}\right)$ e agitação lenta durante 16 horas. O conteúdo de argila foi obtido pelo método da pipeta (GEE; BAUDER, 1986). Os teores de micronutrientes foram determinados através de solução quelante (DTPA). Por meio de espectrofotometria de absorção atômica foram determinados os elementos (Embrapa, 1997). A matéria orgânica foi estimada multiplicando o valor estimado de carbono orgânico por 1,724. Para estimativa do carbono orgânico empregou-se o procedimento de oxidação da matéria orgânica via úmida com dicromato de potássio em meio sulfúrico, empregandose como fonte de energia o calor desprendido do ácido sulfúrico. O excesso de dicromato após a oxidação é titulado com solução padrão de sulfato ferroso amoniacal (Embrapa, 1997).

Os dados da produtividade do milho safra 2012 e soja (2012/2013) foram obtidos por sistema de mapeamento da 
produtividade de grãos de uma colhedora combinada. O sistema é composto por sensor de umidade, sensor de fluxo de grãos, sistema de posicionamento e monitor. Os erros de posicionamento, tempo de enchimento da colhedora e largura da plataforma foram eliminados da base de dados. Para a avaliação da produtividade em cada ponto amostral, calculou-se a média dos 10 registros mais próximos do ponto amostral, obtendo-se assim, 187 registros de produtividade, um para cada ponto da grade amostral.

Para identificação de valores discrepantes no banco de dados, foi utilizada a metodologia proposta por Libardi et al. (1996), calculando-se a dispersão interquartil (DQ), considerando o primeiro (Q1) e o terceiro (Q3) quartil. O limite superior foi definido por (Q3 + 1,5 x DQ) e o inferior por (Q1 - 1,5 x DQ). A normalidade foi testada pelo teste Shapiro-Wilk's $(\mathrm{p}<0,05)$.

Foi utilizado o método dos momentos de Matheron, na avaliação da dependência espacial. Buscou-se ajustar o modelo de semivariância teórico que melhor descreveu a variância empírica dos dados. Os modelos testados forma o esférico, exponencial e o gaussiano. Os ajustes dos modelos foram realizados no programa Vesper. Ajustou-se o modelo que apresentou o maior coeficiente de determinação $\left(R^{2}\right)$ e a menor soma de quadrados do resíduo (SQR). Com base nos modelos ajustados, foram confeccionados os mapas temáticos representativos da variabilidade espacial por meio de interpolação, utilizando krigagem ordinária.

O índice de dependência espacial dos atributos (IDE), foi determinado e classificado, segundo Zimback (2001), utilizando-se a Equação (1)

$$
\mathrm{IDE}=\frac{\mathrm{C}}{\mathrm{C}_{0}+\mathrm{C}} \cdot 100
$$

em que: $C=$ valor da variância espacial; $C_{0}=$ valor do efeito pepita, assumindo os seguintes intervalos: dependência espacial baixa para IDE $<25 \%$, moderada para $25 \% \leq$ IDE $\leq 75 \%$ e forte para IDE $>75 \%$.
A correlação espacial entre os atributos químicos e granulométricos do solo e a produtividade foi realizada por meio do coeficiente de correlação de Pearson. Para a análise de correlação foram selecionados aleatoriamente 30 pontos dos 187 pontos amostrados, pois, quanto maior o número de pontos, maior a chance de rejeição da hipótese de nulidade, sendo 30 um valor adequado de pontos (MONTGOMERY; RUNGER, 2009).

\section{RESULTADOS}

Observou-se que, de 18 atributos do solo avaliados, 12 apresentaram valores discrepantes e estes foram removidos do conjunto de dados, para posterior análise dos mesmos. Os valores discrepantes influenciam na média, amplitude, desvio-padrão e a na distribuição dos dados. Para a produtividade, também foram observados dados discrepantes, provenientes de erro de leitura da largura da plataforma e tempo de enchimento da máquina, no entanto, estes foram eliminados antes da estimativa da produtividade localizada nos pontos amostrais.

Os coeficientes de variação $(\mathrm{CV} \%)$ variaram de 4,36 a $597,54 \%$, para a acidez ativa do solo em $\mathrm{CaCl}_{2}$ e alumínio trocável, respectivamente. Os valores de $\mathrm{CV}$ foram considerados altos $(\mathrm{CV}>35 \%)$ para o fósforo, alumínio e zinco (Tabela 1).

Os valores moderados de CV $(15 \%<\mathrm{CV}<35 \%)$, foram classificados para o potássio, cálcio, magnésio, acidez potencial, cobre, manganês, ferro e areia. O restante dos atributos do solo apresentou CV classificados como baixos $(\mathrm{CV}<15 \%)$.

Os valores médios dos atributos químicos do solo foram classificados segundo a Embrapa (2011) como altos para $\mathrm{Ca}^{+2}, \mathrm{Mg}^{+2}, \mathrm{~K}^{+}, \mathrm{V}, \mathrm{SB}$, matéria orgânica, cobre, ferro, manganês e zinco (Tabela 1). Foram classificados como médios os atributos acidez ativa em $\mathrm{CaCl}_{2}$, fósforo, acidez potencial e capacidade de troca de cátions total, e como baixo para o alumínio. Estes valores já eram esperados pois a área é utilizada para agricultura a 35 anos, sendo corrigida e adubada ao longo deste período.

Tabela 1. Estatística descritiva dos atributos do solo e da produtividade da soja e do milho.

Table 1. Descreptive statiscs of soil attributes, soybean and corn yield.

\begin{tabular}{|c|c|c|c|c|c|c|c|c|c|}
\hline Atributos & Valores Excluídos & Média & Mediana & Min. & Máx. & $\mathrm{CV}^{(7)}(\%)$ & $\mathrm{QI}^{(8)}$ & $\mathrm{QS}^{(9)}$ & $\mathrm{W}^{(10)}$ \\
\hline $\mathrm{pH}^{(1)}$ & 1 & 5,19 & 5,16 & 4,56 & 5,75 & 4,36 & 5,03 & 5,32 & ns \\
\hline $\mathrm{MO}^{(2)}\left(\mathrm{g} \mathrm{kg}^{-1}\right)$ & 2 & 29,12 & 28,40 & 20,21 & 41,50 & 14,11 & 26,08 & 31,79 & $*$ \\
\hline Fósforo $(\mathrm{P})\left(\mathrm{mg} \mathrm{dm}^{-3}\right)$ & 4 & 11,16 & 10,03 & 2,85 & 30,93 & 40,25 & 7,79 & 13,58 & * \\
\hline Potássio $(\mathrm{K})\left(\mathrm{cmol}_{\mathrm{c}} \mathrm{dm}^{-3}\right)$ & 4 & 0,45 & 0,43 & 0,17 & 0,90 & 28,25 & 0,36 & 0,52 & $*$ \\
\hline Cálcio $(\mathrm{Ca})\left(\mathrm{cmol}_{\mathrm{c}} \mathrm{dm}^{-3}\right)$ & 2 & 5,43 & 5,44 & 2,76 & 7,78 & 16,83 & 4,90 & 6,01 & ns \\
\hline Magnésio $(\mathrm{Mg})\left(\mathrm{cmol}_{\mathrm{c}} \mathrm{dm}^{-3}\right)$ & 3 & 1,98 & 1,98 & 1,15 & 3,12 & 18,82 & 1,71 & 2,21 & ns \\
\hline Alumínio $(\mathrm{Al})\left(\mathrm{cmol}_{\mathrm{c}} \mathrm{dm}^{-3}\right)$ & 0 & 0,01 & 0,00 & 0,00 & 0,12 & 597,54 & 0,00 & 0,00 & $*$ \\
\hline $\mathrm{H}+\mathrm{Al}^{(3)}\left(\mathrm{cmol}_{\mathrm{c}} \mathrm{dm}^{-3}\right)$ & 0 & 4,08 & 3,99 & 2,53 & 7,39 & 20,12 & 3,47 & 4,58 & ns \\
\hline $\mathrm{SB}^{(4)}\left(\mathrm{cmol}_{\mathrm{c}} \mathrm{dm}^{-3}\right)$ & 2 & 7,87 & 7,89 & 4,85 & 11,20 & 14,48 & 7,09 & 8,59 & $\mathrm{~ns}$ \\
\hline $\mathrm{T}^{(5)}\left(\mathrm{cmol}_{\mathrm{c}} \mathrm{dm}^{-3}\right)$ & 0 & 11,99 & 11,90 & 9,17 & 14,93 & 9,70 & 11,17 & 12,75 & ns \\
\hline $\mathrm{V}^{(6)}(\%)$ & 3 & 66,17 & 66,00 & 50,07 & 81,59 & 9,22 & 62,86 & 70,54 & ns \\
\hline Cobre $(\mathrm{Cu})\left(\mathrm{mg} \mathrm{dm}^{-3}\right)$ & 3 & 7,59 & 7,32 & 3,94 & 12,11 & 22,87 & 6,25 & 8,73 & $\mathrm{~ns}$ \\
\hline Manganês $(\mathrm{Mn})\left(\mathrm{mg} \mathrm{dm}^{-3}\right)$ & 5 & 61,20 & 58,73 & 27,63 & 104,68 & 24,60 & 50,87 & 72,00 & ns \\
\hline Ferro $\left(\mathrm{Fe}\left(\mathrm{mg} \mathrm{dm}^{-3}\right)\right.$ & 1 & 49,60 & 47,61 & 18,36 & 92,59 & 31,66 & 37,41 & 59,86 & $*$ \\
\hline Zinco $(\mathrm{Zn})\left(\mathrm{mg} \mathrm{dm}^{-3}\right)$ & 0 & 4,79 & 4,52 & 1,28 & 13,08 & 35,64 & 3,77 & 5,34 & $*$ \\
\hline Argila $\left(\mathrm{g} \mathrm{kg}^{-1}\right)$ & 0 & 540,11 & 550,00 & 450,00 & 650,00 & 11,64 & 500,00 & 600,00 & $*$ \\
\hline Silte $\left(\mathrm{g} \mathrm{kg}^{-1}\right)$ & 2 & 165,00 & 150,00 & 125,00 & 200,00 & 12,36 & 150,00 & 175,00 & * \\
\hline Areia $\left(\mathrm{g} \mathrm{kg}^{-1}\right)$ & 0 & 294,25 & 275,00 & 200,00 & 400,00 & 22,18 & 250,00 & 350,00 & $*$ \\
\hline Produtividade soja $\left(\mathrm{kg} \mathrm{ha}^{-1}\right)$ & 0 & 3676,3 & 3674,8 & 3023,7 & 4387,2 & 8,38 & 3439,8 & 3907,1 & ns \\
\hline Produtividade milho $\left(\mathrm{kg} \mathrm{ha}^{-1}\right)$ & 0 & 5421,4 & 5497,2 & 3189,8 & 6610,4 & 10,88 & 5219,2 & 5779,5 & ns \\
\hline
\end{tabular}


Os atributos cálcio, alumínio, acidez trocável, soma de bases, ferro e zinco não apresentaram dependência espacial (Tabela 2). A falta de dependência espacial ocorre quando o valor da semivariância é igual ao patamar, em qualquer distância entre os pares de pontos, sendo chamado de efeito pepita puro. O efeito pepita puro ocorre quando a distribuição da variável na área é aleatória, ou quando distância mínima entre os pontos amostrais é superior à distância da dependência espacial.

Para o restante dos atributos do solo, os variogramas obtidos apresentaram-se bem ajustados, com valores do coeficiente de determinação $\left(\mathrm{R}^{2}\right)$ acima de 0,77 e os menores valores de soma de quadrados dos resíduos. O alcance da dependência espacial variou 122 a $2362 \mathrm{~m}$, para o potássio e o magnésio, respectivamente. Para os teores de potássio, magnésio, argila e areia foi observada forte dependência espacial (IDE > 0,75) e para os demais atributos químicos do solo foi encontrada moderada dependência espacial $(0,25 \leq$ IDE $\leq 0,75)$, sendo o maior IDE (96) observado para a areia e o menor (50) para a capacidade de trocas de cargas, saturação por bases, manganês e silte.

A produtividade da soja apresentou dependência espacial com alcance de $905 \mathrm{~m}$. O modelo teórico que melhor se ajustou foi o esférico com coeficiente de determinação de 0,99 e forte dependência espacial (87\%), para a produtividade do milho foi observada moderada dependência espacial (63\%) com alcance de $157 \mathrm{~m}$ e coeficiente de determinação de 0,91 do modelo teórico exponencial (Tabela 2).

Tabela 2. Parâmetros dos modelos teóricos de semivariância ajustados para os atributos físicos e químicos do solo e produtividade da soja. Table 2. Parameters of the theoretical models of semi-variance adjusted for the physical and chemical attributes of the soil and soybean yield.

\begin{tabular}{|c|c|c|c|c|c|c|c|}
\hline Atributos & Modelo & Alcance & $\mathrm{C}_{0}+\mathrm{C}^{(7)}$ & $\mathrm{C}_{0}(8)$ & $\operatorname{IDE}(9)$ & SQR $(10)$ & $\mathrm{R}^{2(11)}$ \\
\hline $\mathrm{pH}^{(1)}$ & $\mathrm{ESF}^{(12)}$ & 361 & 0,058 & 0,020 & 64 & $4,4 x \cdot 10^{-5}$ & 0,93 \\
\hline $\mathrm{MO}^{(2)}\left(\mathrm{g} \mathrm{kg}^{-1}\right)$ & GAU(13) & 509 & 24,46 & 8,29 & 66 & 0,4 & 0,99 \\
\hline Fósforo $(\mathrm{P})\left(\mathrm{mg} \mathrm{dm}^{-3}\right)$ & $\operatorname{EXP}^{(14)}$ & 500 & 26,02 & 10,72 & 59 & 1,6 & 0,98 \\
\hline Potássio $(\mathrm{K})\left(\mathrm{cmol}_{\mathrm{c}} \mathrm{dm}^{-3}\right)$ & EXP & 122 & 0,0200 & 0,0036 & 82 & $1,9 \times 10^{-6}$ & 0,96 \\
\hline Cálcio (Ca) $\left(\mathrm{cmol}_{\mathrm{c}} \mathrm{dm}^{-3}\right)$ & $\mathrm{EPP}^{(15)}$ & - & 0,8025 & 0,8025 & - & - & - \\
\hline Magnésio $(\mathrm{Mg})\left(\mathrm{cmol}_{\mathrm{c}} \mathrm{dm}^{-3}\right)$ & GAU & 2362 & 0,523 & 0,103 & 80 & $2,0 \times 10^{-3}$ & 0,91 \\
\hline Alumínio (Al) $\left(\mathrm{cmol}_{\mathrm{c}} \mathrm{dm}^{-3}\right)$ & EPP & - & 0,002 & 0,002 & - & - & - \\
\hline $\mathrm{H}+\mathrm{Al}^{(3)}\left(\mathrm{cmol}_{\mathrm{c}} \mathrm{dm}^{-3}\right)$ & EPP & - & 0,9019 & 0,9019 & - & - & - \\
\hline $\mathrm{SB}^{(4)}\left(\mathrm{cmol}_{\mathrm{c}} \mathrm{dm}^{-3}\right)$ & EPP & - & 1,3690 & 1,3690 & - & - & - \\
\hline $\mathrm{T}^{(5)}\left(\mathrm{cmol}_{\mathrm{c}} \mathrm{dm}^{-3}\right)$ & EXP & 195 & 1,403 & 0,701 & 50 & 0,0457 & 0,77 \\
\hline $\mathrm{V}^{(6)}(\%)$ & ESF & 286 & 40,03 & 20,01 & 50 & 4,46 & 0,97 \\
\hline Cobre $(\mathrm{Cu})\left(\mathrm{mg} \mathrm{dm}^{-3}\right)$ & ESF & 580 & 3,355 & 1,485 & 56 & 0,0574 & 0,97 \\
\hline Manganês (Mn) $\left(\mathrm{mg} \mathrm{dm}^{-3}\right)$ & EXP & 255 & 251,90 & 125,90 & 50 & 874,00 & 0,88 \\
\hline Ferro $\left(\mathrm{Fe}\left(\mathrm{mg} \mathrm{dm}^{-3}\right)\right.$ & EPP & - & 246,91 & 246,91 & - & - & - \\
\hline Zinco $(\mathrm{Zn})\left(\mathrm{mg} \mathrm{dm}^{-3}\right)$ & EPP & - & 2,1307 & 2,1307 & - & - & - \\
\hline Argila $\left(\mathrm{g} \mathrm{kg}^{-1}\right)$ & GAU & 431 & 6630,0 & 260,0 & 94 & 139711 & 0,99 \\
\hline Silte $\left(\mathrm{g} \mathrm{kg}^{-1}\right)$ & EXP & 300 & 412,90 & 206,04 & 50 & 1208,0 & 0,92 \\
\hline Areia $\left(\mathrm{g} \mathrm{kg}^{-1}\right)$ & GAU & 418 & 7587,00 & 240,00 & 96 & 250023 & 0,99 \\
\hline Produtividade soja $\left(\mathrm{kg} \mathrm{ha}^{-1}\right)$ & ESF & 905 & 86090 & 11000 & 87 & $2,1 \times 10^{7}$ & 0,99 \\
\hline Produtividade milho $\left(\mathrm{kg} \mathrm{ha}^{-1}\right)$ & EXP & 213 & 227000 & 27400 & 88 & $4,4 \times 10^{8}$ & 0,88 \\
\hline
\end{tabular}

(1)Acidez ativa em $\mathrm{CaCl}_{2}$; ${ }^{(2)}$ Matéria orgânica; (3) Acidez potencial; (4)Soma de bases; (5)Capacidade de trocas de cátions; (6)Saturação por bases; (7)Patamar; ${ }^{(8)}$ Efeito pepita; (9) Índice de dependência espacial; (10)Soma de quadrados do resíduo; ${ }^{(11)}$ Coeficiente de determinação; (12)Esférico; (13) Gaussiano; (14)Exponencial; (15)Efeito pepita puro.

Pela validação cruzada dos dados (Tabela 3) observa-se que as melhores estimativas em locais não amostrados foram obtidas em ordem decrescente para a: argila, areia, matéria orgânica, cobre, acidez ativa, fósforo, potássio, silte, saturação por bases, manganês, magnésio e capacidade de troca de cátions total.

Para a acidez ativa, matéria orgânica, fósforo, potássio, cobre, argila e areia os coeficientes de regressão e os erros quadráticos estão próximos de 1 e 0 , respectivamente. Assim, considera-se as estimativas como satisfatórias, entretanto, os coeficientes de determinação da validação cruzada segundo a classificação adotada por Nanni et al. (2011) foram considerados como excelentes $\left(\mathrm{R}^{2}>0,75\right)$ para a argila e areia e baixos $\left(\mathrm{R}^{2}<0,50\right)$ para o restante dos atributos testados. Bottega et al. (2013a) e Nanni et al. (2011) também observaram baixos coeficientes de determinação da validação cruzada para todos os atributos químicos do solo estudados.

Os coeficientes de correlação de Pearson entre a produtividade do milho e da soja e os atributos do solo
(Tabela 4), foram significativos tanto a $1 \%$ quanto a $5 \%$ de probabilidade, sendo consideradas as correlações (r) moderadas $(0,29<\mathrm{r}<0,50)$ e fortes $(\mathrm{r}>0,50)$ segundo a classificação proposta por Cohen (1988). Foi observada correlação significativa positiva forte $(0,51)$ e moderada $(0,49)$ entre a produtividade da soja e os teores de fósforo e magnésio, respectivamente, e moderada negativa com o silte $(-0,39)$. Desta forma podemos considerar que quando os atributos químicos se encontram em níveis preconizados para as culturas, a sua correlação com a produtividade é minimizada e outros atributos passam a explicar a variabilidade na produção.

Não foi observado correlação significativa entre a produtividade do milho e os atributos do solo avaliados (Tabela 4). Provavelmente isto ocorreu pela interferência causada pelo ataque dos porcos no talhão. Os ataques ocorreram em manchas pontuais em toda a extensão do talhão, interferindo na coleta dos dados de produtividade da colhedora. 
Tabela 3. Parâmetros da validação cruzada dos modelos teóricos de semivariância ajustados para os atributos químicos e físicos do solo e da produtividade da soja.

Table 3. Cross-validation parameters of the theoretical models of semivariance adjusted for the chemical and physical soil attributes and soybean yield.

\begin{tabular}{|c|c|c|c|c|}
\hline Atributos & Coeficiente de regressão & Intercepto $(\mathrm{Y})$ & Erro quadrático (SE) & $\mathrm{R}^{2(5)}$ \\
\hline $\mathrm{pH}^{(1)}$ & 1,017 & $-0,08$ & 0,104 & 0,34 \\
\hline $\mathrm{MO}(2)$ & 1,034 & $-0,98$ & 0,079 & 0,48 \\
\hline $\mathrm{P}\left(\mathrm{mg} \mathrm{dm^{-3 } )}\right.$ & 0,954 & 0,48 & 0,105 & 0,31 \\
\hline $\mathrm{K}\left(\mathrm{cmol}_{\mathrm{c}} \mathrm{dm}^{-3}\right)$ & 0,916 & 0,04 & 0,102 & 0,31 \\
\hline $\mathrm{Mg}\left(\mathrm{cmol}_{\mathrm{c}} \mathrm{dm}^{-3}\right)$ & 0,754 & 0,48 & 0,143 & 0,13 \\
\hline $\mathrm{T}^{(3)}\left(\mathrm{cmol}_{\mathrm{c}} \mathrm{dm}^{-3}\right)$ & 0,734 & 3,19 & 0,139 & 0,13 \\
\hline $\mathrm{V}^{(4)}(\%)$ & 0,872 & 8,53 & 0,135 & 0,19 \\
\hline $\mathrm{Cu}\left(\mathrm{mg} \mathrm{dm} \mathrm{m}^{-3}\right)$ & 0,990 & 0,08 & 0,099 & 0,35 \\
\hline $\mathrm{Mn}\left(\mathrm{mg} \mathrm{dm}^{-3}\right)$ & 0,885 & 7,21 & 0,141 & 0,18 \\
\hline Argila $\left(\mathrm{g} \mathrm{kg}^{-1}\right)$ & 1,037 & $-20,74$ & 0,023 & 0,92 \\
\hline Silte $\left(\mathrm{g} \mathrm{kg}^{-1}\right)$ & 0,867 & 21,96 & 0,128 & 0,20 \\
\hline Areia $\left(\mathrm{g} \mathrm{kg}^{-1}\right)$ & 1,033 & $-9,07$ & 0,023 & 0,92 \\
\hline Produtividade soja $\left(\mathrm{kg} \mathrm{ha}^{-1}\right)$ & 1,018 & $-65,84$ & 0,038 & 0,79 \\
\hline Produtividade milho $\left(\mathrm{kg} \mathrm{ha}^{-1}\right)$ & 0,884 & 630,91 & 0,134 & 0,19 \\
\hline
\end{tabular}

${ }^{(1)}$ Acidez ativa em CaCl2; ${ }^{(2)}$ Matéria orgânica; ${ }^{(3)}$ Soma de bases; ${ }^{(4)}$ Capacidade de trocas de cátions; ${ }^{(5)}$ Coeficiente de determinação.

Tabela 4. Coeficiente de correlação de Pearson ( $r$ ) entre a produtividade da soja e do milho e os atributos químicos e físicos do solo. Table 4. Pearson correlation coefficient ( $r$ ) between soybean and corn yield with chemical and physical soil attributes.

\begin{tabular}{|c|c|c|c|c|c|}
\hline \multirow{2}{*}{ Variável } & \multicolumn{2}{|c|}{$\mathrm{r}$} & \multirow{2}{*}{ Variável } & \multicolumn{2}{|c|}{$\mathrm{r}$} \\
\hline & Soja & Milho & & Soja & Milho \\
\hline $\mathrm{P}\left(\mathrm{mg} \mathrm{dm}^{-3}\right)$ & $0,51^{* *}$ & 0,04 & $\mathrm{~K}\left(\mathrm{cmol}_{\mathrm{c}} \mathrm{dm}^{-3}\right)$ & 0,04 & 0,02 \\
\hline $\mathrm{Mg}\left(\mathrm{cmol}_{\mathrm{c}} \mathrm{dm}^{-3}\right)$ & $0,49^{* *}$ & $-0,02$ & $\mathrm{pH}$ & 0,02 & $-0,12$ \\
\hline Argila $\left(\mathrm{g} \mathrm{kg}^{-1}\right)$ & 0,36 & 0,34 & $\mathrm{Al}\left(\mathrm{cmol}_{\mathrm{c}} \mathrm{dm}^{-3}\right)$ & $-0,01$ & 0,03 \\
\hline $\mathrm{MO}\left(\mathrm{g} \mathrm{kg}^{-1}\right)$ & 0,33 & 0,28 & $\mathrm{Zn}\left(\mathrm{mg} \mathrm{dm}^{-3}\right)$ & $-0,02$ & 0,10 \\
\hline $\mathrm{SB}\left(\mathrm{cmol}_{\mathrm{c}} \mathrm{dm}^{-3}\right)$ & 0,24 & 0,11 & $\mathrm{H}+\mathrm{Al}\left(\mathrm{cmol}_{\mathrm{c}} \mathrm{dm}^{-3}\right)$ & $-0,07$ & 0,09 \\
\hline V $(\%)$ & 0,14 & $-0,03$ & $\mathrm{Cu}\left(\mathrm{mg} \mathrm{dm}^{-3}\right)$ & $-0,13$ & $-0,14$ \\
\hline $\mathrm{T}\left(\mathrm{cmol}_{\mathrm{c}} \mathrm{dm}^{-3}\right)$ & 0,14 & 0,18 & $\mathrm{Fe}\left(\mathrm{mg} \mathrm{dm}^{-3}\right)$ & $-0,17$ & $-0,01$ \\
\hline $\operatorname{Mn}\left(\mathrm{mg} \mathrm{dm}^{-3}\right)$ & 0,11 & 0,04 & Areia $\left(\mathrm{g} \mathrm{kg}^{-1}\right)$ & $-0,19$ & $-0,31$ \\
\hline $\mathrm{Ca}\left(\mathrm{cmol}_{\mathrm{c}} \mathrm{dm}^{-3}\right)$ & 0,05 & 0,14 & Silte $\left(\mathrm{g} \mathrm{kg}^{-1}\right)$ & $-0,39^{*}$ & 0,00 \\
\hline
\end{tabular}

(*) Significância ao nível de $5 \%$ de probabilidade; ${ }^{* *}$ Significância de $1 \%$ de probabilidade.

Na Figura 2 estão apresentados os mapas temáticos dos atributos químicos e físicos do solo elaborados pela interpolação dos valores amostrados utilizando a técnica da krigagem ordinária.

Observa-se que os mapas da argila e areia apresentam maior continuidade espacial, isso se deve ao excelente ajuste do $\mathrm{R}^{2}$ da validação cruzada para estes atributos do solo, além disso, observa-se similaridade na localização entre os maiores valores de argila e os menores valores areia. Este comportamento é justificado pois as frações do solo geralmente apresentam comportamento inverso, principalmente de distribuição, pois como são medidos em porcentagem quando há acréscimo de um ocorre redução de outro (SOUZA et al., 2004).

\section{DISCUSSÃO}

A alta dispersão relativa para o $\mathrm{P}$ e Al, moderada para o $\mathrm{K}, \mathrm{Ca}, \mathrm{Mg}, \mathrm{H}+\mathrm{Al}$ e baixa para o $\mathrm{pH}, \mathrm{T}$ e argila, corroboram com estudos anteriores em Latossolos (Bottega et al., 2013a; Sana et al., 2014; Santi et al., 2012). A ampla maioria dos estudos de variabilidade espacial de atributos químicos do solo classificam o fósforo como de alta variabilidade. Este fato pode ser decorrente de sucessivas adubações com este nutriente no sulco de semeadura, o que acarreta grandes variações a curtas distâncias (CHERUBIN et al., 2015; NANNI et al., 2011).
Também se observou alta dispersão relativa para o $\mathrm{Zn}$, que é um micronutriente que se encontra originalmente em baixos teores no solo. A dispersão relativa foi moderada para os micronutrientes ( $\mathrm{Fe}, \mathrm{Mn}$ e $\mathrm{Cu}$ ) que normalmente apresentam altos teores nos Latossolos argilosos e para os macronutrientes $\mathrm{Ca}, \mathrm{Mg}$ e $\mathrm{K}$ que são aplicados em área total a lanço.

A dispersão relativa da produtividade soja e do milho foi classificada como baixa $(\mathrm{CV}<15 \%)$ e corrobora com os valores encontrados por Bottega et al. (2013b) que em um estudo de três safras consecutivas de soja também observaram coeficientes de variação abaixo dos 15\%. Amado et al. (2007) estudando a variabilidade espacial de diferentes safras encontraram valores de dispersão relativa classificados de baixa a moderada variabilidade (12\% a 35\%). Segundo Amado et al. (2007), as menores dispersões relativas são observadas em anos com precipitação pluviométrica normais.

Amado et al. (2007) e Bottega et al. (2013b), encontraram em seus estudos, dependência espacial para a produtividade da soja e milho com alcances variando de 56,1 a $764 \mathrm{~m}$, e IDE variando de moderado a forte. Índices de dependência espacial moderados e fortes indicam que os variogramas explicam a maior parte da variância dos dados. Atributos que possuem forte dependência espacial são influenciados por fatores intrínsecos do solo, já os que possuem fraca dependência espacial receberam maior influência de fatores 
externos, devido a aplicações de corretivos e fertilizantes no cultivo do solo (SOUZA et al., 2010).

Kerry; Oliver (2004) propuseram a utilização de distâncias máximas entre as amostras não superiores à metade do valor do alcance da dependência espacial obtido. Isso porque, este parâmetro indica a distância a que os pontos amostrados estão correlacionados (RODRIGUES et al., 2012; AQUINO et al., 2014). Neste estudo, entretanto, para todos os atributos do solo que apresentam dependência espacial esta recomendação foi atendida, e mesmo assim não se observou boas estimativas em locais não amostrados.

Diversos autores atribuem essa baixa qualidade das estimativas ao uso de malhas amostrais insuficientes para captarem a variabilidade horizontal do solo e recomendaram o uso de malhas amostrais mais densas (CHERUBIN et al. 2014; NANNI et al. 2011). Entretanto, deve-se considerar também o período em que a área em estudo está sendo utilizada para agricultura, e as alterações intensas na variabilidade dos atributos químicos do solo causadas pelas operações de adubação e correção do solo realizadas ao longo do tempo. Tais práticas podem influenciar de tal forma que seja inviável ou até impossível modelar a variabilidade com bons ajustes da validação cruzada em função das profundas alterações ocasionadas por fatores extrínsecos.

A estimativa da produtividade da soja foi considerada excelente, com $\mathrm{R}^{2}$ da validação cruzada de 0,79 , coeficiente de regressão e erro quadrático próximos de 1 e 0 , respectivamente. Amado et al. (2007) concluíram que a soja apresenta, em anos com precipitação normal, restrita capacidade de discriminar a variabilidade espacial existente na lavoura. Porém, neste trabalho, mesmo em ano sem déficit hídrico, observou-se que a produtividade da soja apresentou forte dependência espacial.
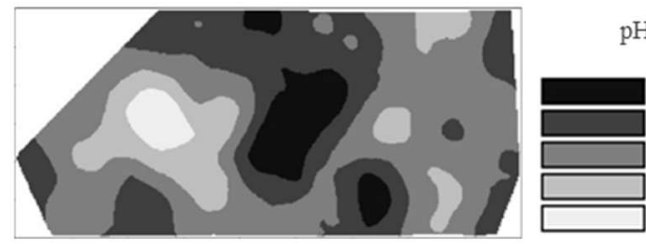

$\mathrm{pH}$
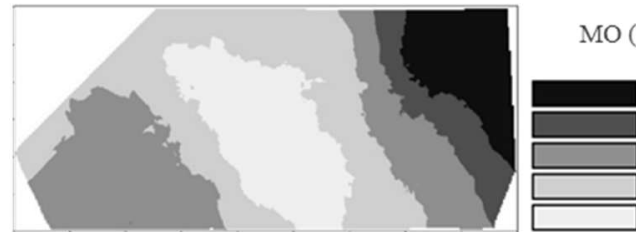

$\mathrm{OO}\left(\mathrm{g} \mathrm{kg}^{-1}\right)$
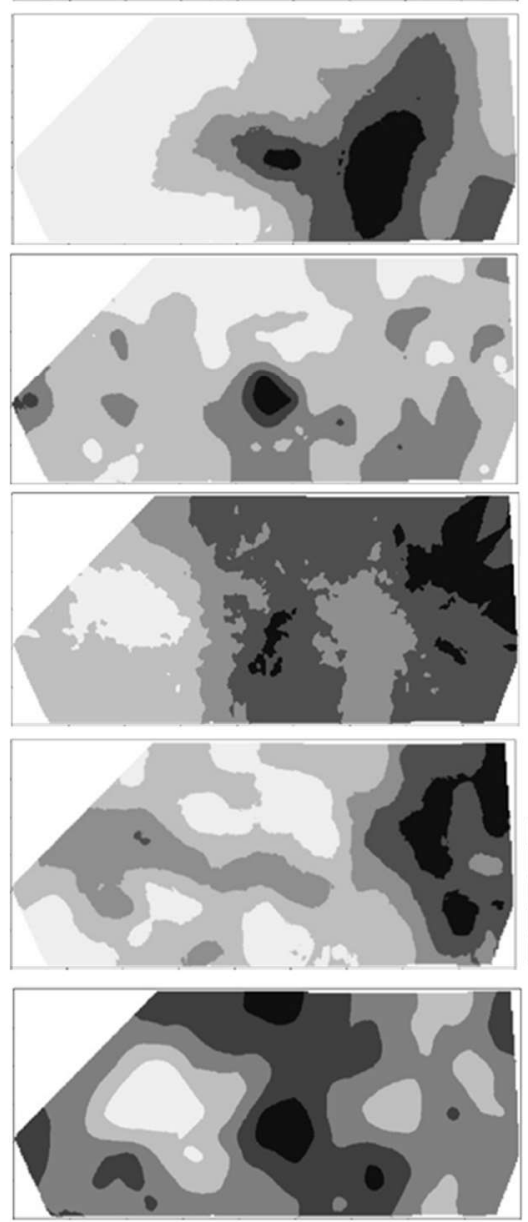

$\mathrm{P}(\mathrm{mg} \mathrm{dm}-3)$
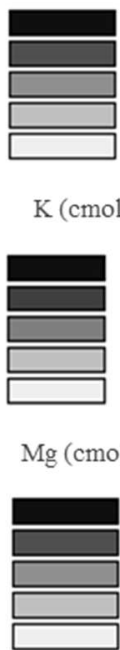

$\mathrm{T}\left(\mathrm{cmol}_{\mathrm{c}} \mathrm{dm}^{-3}\right)$

$\mathrm{V}(\%)$

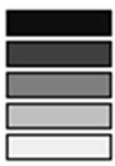

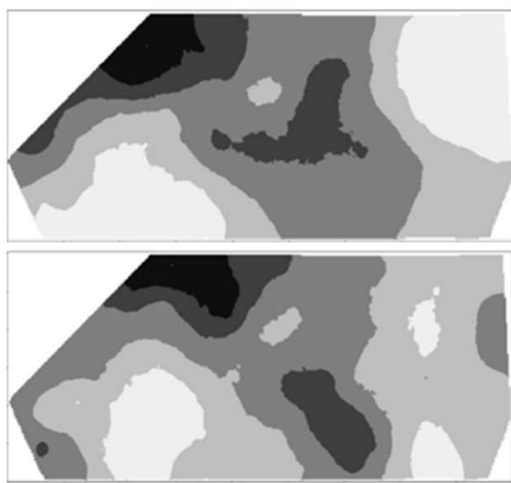

$>15.4$

$>13.3$

$>11.3$ $>9.3$ $>7.2$

$\mathrm{K}\left(\mathrm{cmol}_{\mathrm{c}} \mathrm{dm}^{-3}\right)$

$>0.782$ $>0.644$ $>0.506$ $>0.368$ $>0.230$

$\mathrm{Mg}\left(\mathrm{cmol}_{\mathrm{c}} \mathrm{dm}^{-3}\right)$

$>2.18$

$>2.02$

$>1.85$

$>1.69$

$>1.53$

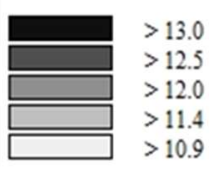

$>71.3$

$>67.6$

$>63.9$

$>60.2$

$>56.6$
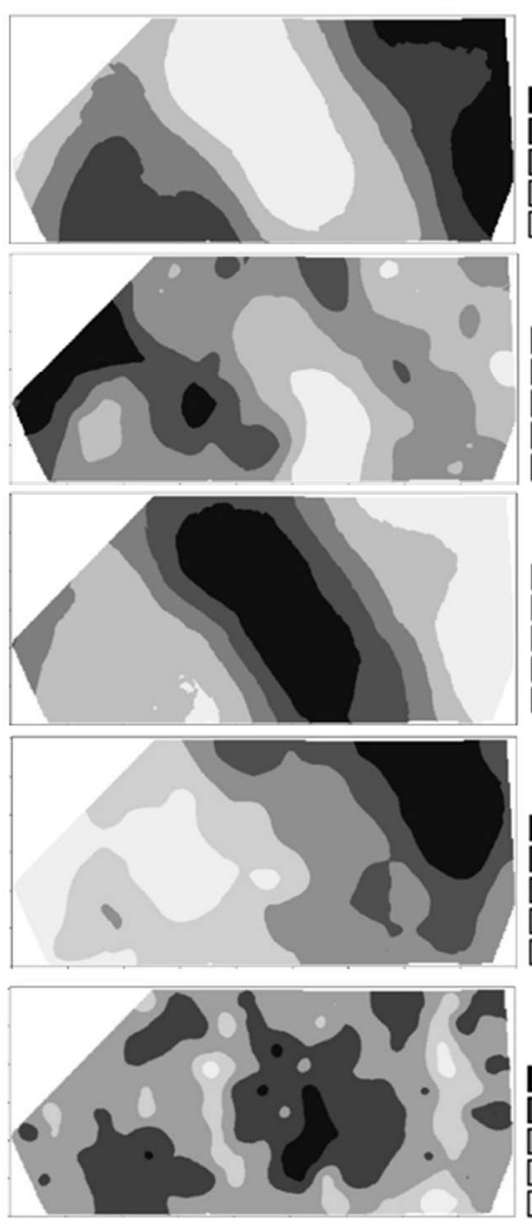

$\mathrm{Cu}\left(\mathrm{mg} \mathrm{dm}^{-3}\right)$

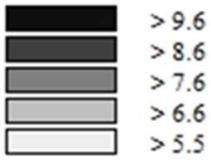

$\operatorname{Mn}\left(\mathrm{mg} \mathrm{dm}{ }^{-3}\right)$

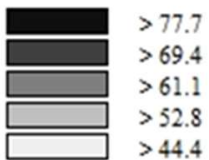

Argila $\left(\mathrm{g} \mathrm{kg}^{-1}\right)$

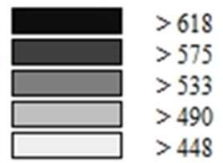

Silte $\left(\mathrm{g} \mathrm{kg}^{-1}\right)$

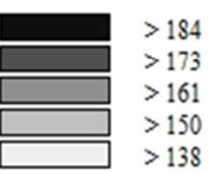

Areia $\left(\mathrm{g} \mathrm{kg}^{-1}\right)$

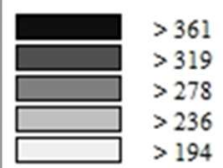

Produtividade

soja $\left(\mathrm{kg} \mathrm{ha}^{-1}\right)$

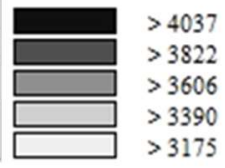

Produtividade milho (kg ha-1)

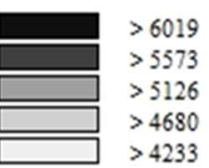

Figura 2. Mapas temáticos de distribuição espacial dos atributos do solo e da produtividade de soja e milho.

Figure 2. Thematic maps of spatial distribution of soil attributes and soybean and corn yield. 
Para a estimativa da produtividade do milho, o $\mathrm{R}^{2}$ foi baixo $(0,19)$, contrariando os resultados encontrados por Amado et al. (2007). Provavelmente isto ocorreu, devido a grande população de porcos selvagens existente na região, que percorrem toda a extensão do talhão quando este é cultivado por uma cultura atrativa como o milho, ocasionando perdas de produtividade localizadas e aleatórias que interferem na variabilidade da produtividade do milho.

A correlação positiva entre a produtividade da soja e o $\mathrm{P}$ e o $\mathrm{Mg}$ no solo é esperado uma vez que originalmente os Latossolos apresentam baixa disponibilidade de P e bases trocáveis. Nos solos da região do Cerrado em condição natural, o teor de P é muito baixo; no entanto, nos solos cultivados a variação pode ser grande em razão da alta capacidade de retenção do P na fase sólida e da sua aplicação na linha de semeadura (SANA et al., 2014).

A moderada correlação negativa obtida entre o silte e a produtividade da soja pode estar associada a formação de crosta superficial, o que prejudicaria o desenvolvimento inicial das plântulas e diminuiria a infiltração de água. $\mathrm{O}$ desenvolvimento de crostas superficiais no solo é originado a partir da quebra dos agregados e dispersão das partículas, com influência da textura do solo (HU et al., 2012), onde as partículas do solo são rearranjadas e consolidadas em uma estrutura coesa, reduzindo o número e a continuidade do espaço poroso, afetando a infiltração e a distribuição de água ao logo do perfil do solo (ZONTA et al., 2012; ARMENISE et al., 2018).

Destaca-se que a não existência de correlações entre a acidez ativa do solo e os teores de alumínio com produtividade. Este fato é explicado pois a maioria dos pontos amostrais apresentaram valores de $\mathrm{pH}$ adequados ao cultivo de soja e milho (EMBRAPA, 2011). Com relação aos teores de alumínio, somente sete das 187 amostras apresentaram este elemento em baixas concentrações.

Comparando os mapas da argila e MO, observa-se uma similaridade na localização dos maiores teores de argila com os maiores de MO. Este comportamento se deve provavelmente a capacidade da argila em reter e proteger a decomposição da MO. Para o mapa do silte e do restante dos atributos químicos do solo podemos observar menor continuidade espacial e a ocorrência de manchas, isto se deve a menor precisão das estimativas de valores em regiões não amostradas, podendo ser confirmado analisando os menores valores dos parâmetros de ajuste da validação cruzada.

\section{CONCLUSÕES}

Não foi detectada dependência espacial para os atributos cálcio, alumínio, acidez potencial, soma de bases, ferro e zinco.

Os melhores ajustes dos variogramas e da validação cruzada foram obtidos para os atributos físicos areia e argila.

A produtividade da soja apresentou forte dependência espacial e se correlacionou positivamente de forma forte com o fósforo e moderada com o magnésio.

\section{AGRADECIMENTOS}

Ao Sr. Florino Wielemaker pela colaboração e cessão da área estudada, ao CNPQ pela concessão da bolsa de estudos, a Danilo Gomes Fortes, Eduardo Messias de Andrade Almeida, Eliéser de Almeida e Matheus Andrade Martinez pelo auxílio na condução dos trabalhos de campo.

\section{REFERÊNCIAS}

ALVARES, C. A.; STAPE, J. L.; SENTELHAS, P. C.; GONÇALVES, J. L. M.; SPAROVEK, G. Köppen's climate classification map for Brazil. Meteorologische Zeitschrift, v. 22, n. 6, p. 711-728, 2013. DOI: https://doi.org/10.1127/0941-2948/2013/0507

AMADO, T. J. C.; PONTELLI, C. B.; SANTI, A. L.; VIANA, J. H. M.; SULZSBACH, L. A. S. Variabilidade espacial e temporal da produtividade de cultura sob sistema plantio direto. Pesquisa Agropecuária Brasileira, v. 42, n. 8, p. 1101-1110, 2007. DOI: https://doi.org/10.1590/S0100-204X2007000800006

AQUINO, R. E.; CAMPOS, M. C. C.; MARQUES JÚNIOR, J.; OLIVEIRA, I. A. de; MANTOVANELI, B. C.; SOARES, M. D. R. Geostatistics in assessment of physical properties in a latossolo (oxisol) under native forest and grassland in Manicoré, Amazonas, Brazil. Revista Brasileira de Ciência do Solo, v. 38, n. 2, p. 397-406, 2014. DOI: https://doi.org/10.1590/S010006832014000200004

ARAÚJO, D. C. dos S.; MONTENEGRO, S. M. G. L.; MONTENEGRO, A. A. de A.; SILVA JUNIOR, V. DE P.; SANTOS, S. M. dos. Spatial variability of soil attributes in an experimental basinin the semi-arid region of Pernambuco, Brazil. Revista Brasileira de Engenharia Agrícola e Ambiental, v. 22, n. 1, p. 38-44, 2018. DOI: http://dx.doi.org/10.1590/18071929/agriambi.v22n1p38-44

ARMENISE, E.; SIMMONS, R. W.; AHN, S.; GARBOUT, A.; DOERR, S. H.; MOONEY, S. J.; STURROCK, C. J.; RITZ, K. Soil seal development under simulated rainfall: structural, physical and hydrological dynamics. Journal of Hydrology, v. 556, p. 211-219, 2018. DOI: https://doi.org/10.1016/j.jhydrol.2017.10.073

BAERDEMAEKER, J. de. Precision Agriculture Technology and Robotics for Good Agricultural Practices. IFAC Proceedings Volumes, v. 46, n. 4, p. 14, 2013. DOI: https://doi.org/10.3182/20130327-3-JP3017.00003

BOTTEGA, E. L.; QUEIROZ, D. M.; PINTO, F. A. C.; SOUZA, C. M. A. Variabilidade espacial de atributos do solo em sistema de semeadura direta com rotação de culturas no cerrado brasileiro. Revista Ciência Agronômica, v. 44, n. 1, p. 1-9, 2013a.

BOTTEGA, E. L.; PINTO, F. A. C.; QUEIROZ, D. M.; SANTOS, N. T.; SOUZA, C. M. A. (2013b) Variabilidade espacial e temporal da produtividade de soja no Cerrado brasileiro. Revista Agrarian, v. 6, n. 20, p. 167-177, 2013b.

CERRI, D. G. P.; MAGALHÃES, P. S. G. Correlation of physical and chemical attributes of soil with sugarcane yield. Pesquisa Agropecuária Brasileira, v. 47, n. 4, p. 613-620, 2012. DOI: https://doi.org/10.1590/S0100204X2012000400018

CHERUBIN, M. R.; SANTI, A. L.; EITELWEIN, M. T.; MENEGOL, D. R.; DA ROS, C. O.; PIAS, O. H. C.; BERGHETTI, J. Eficiência de malhas amostrais utilizadas na caracterização da variabilidade espacial de fósforo e potássio. Ciência Rural, v. 44, n. 3, p. 425-432, 2014. DOI: https://doi.org/10.1590/S010384782014000300007

CHERUBIN, M. R.; SANTI, A. L.; EITELWEIN, M. T.; AMADO, T. J. C.; SIMON, D. H.; DAMIAN, J. M. 
Dimensão da malha amostral para caracterização da variabilidade espacial de fósforo e potássio em Latossolo Vermelho. Pesquisa Agropecuária Brasileira, v. 50, n. 2, p. 168-177, $2015 . \quad$ DOI: https://doi.org/10.1590/S0100-204X2015000200009

COHEN, J. Statistical power analysis for the behavioral sciences. 2 ed. Hilsdale. NJ: Lawrence Earlbaum Associates, 1988. 579p.

CRUZ, J. S.; ASSIS JÚNIOR, R. N. DE; MATIAS, S. S. R.; CAMACHO-TAMAYO, J. H. Spatial variability of an Alfisol cultivated with sugarcane. Ciencia e Investigación Agraria, v. 38, n. 1, p. 155-164, 2011. DOI: 16202011000100015

EMBRAPA_Empresa Brasileira de Pesquisa Agropecuária. Manual de métodos de análise de solo. 2. ed. rev. atual. Rio de Janeiro: Centro Nacional de Pesquisa de Solos, 1997. 212p.

EMBRAPA_Empresa Brasileira de Pesquisa Agropecuária. Tecnologias de produção de soja - região central do Brasil - 2012 e 2013. Londrina: Embrapa Soja, 2011. 261p.

EMBRAPA_Empresa Brasileira de Pesquisa Agropecuária. Sistema brasileiro de classificação de solos. 3 ed. Brasília: Embrapa Informação Tecnológica, 2013. 353p.

GEE, G. W.; BAUDER, W. Particle-size analysis. In: BLACK C. A. (Ed.) Methods of soil analysis. Part 1. Madison, American Society of Agronomy, 1986. (Agronomy, 9)

GUO, W.; MAAS, S. J.; BRONSON, K. F. Relationship between cotton yield and soil electrical conductivity, topography, and landsat imagery. Precision Agriculture, $\begin{array}{llll}\text { v. } 13, \quad \text { p. } 678-692, & 2012 . & \text { DOI: }\end{array}$ https://doi.org/10.1007/s11119-012-9277-2

HU, X.; LIU, L. Y.; LI, S. J.; CAI, Q. G.; LÜ, Y. L.; GUO, J. R. Development of soil crusts under simulated rainfall and crust formation on a loess soil as influenced by polyacrylamide. Pedosphere, v. 22, n. 3, p. 415-424, 2012. DOI: https://doi.org/10.1016/S10020160(12)60027-7

KERRY, R.; OLIVER, M. Average variograms to guide soil sampling. International Journal of Applied Earth Observation and Geoinformation, v. 5, n. 4, p. 307325. 2004.

DOI: https://doi.org/10.1016/j.jag.2004.07.005

LIBARDI, P. L.; MANFRON, P. A.; MORAES, S. O.; TUON, R. L. Variabilidade da umidade gravimétrica de um solo hidromórfico. Revista Brasileira de Ciência do Solo, v. 20, n. 1, p. 1-12, 1996.

MONTGOMERY, D. C.; RUNGER, G. C. Estatística aplicada e probabilidade para engenheiros. 4 ed. Rio de Janeiro: Editora LTC, 2009. 493p.

NANNI, M. R.; POVH, F. P.; DEMATTÊ, J. A. M.; OLIVEIRA, R. B. de; CHICATI, M. L.; CEZAR, E. Optimum size in grid soil sampling for variable rate application in site-specific management. Scientia Agricola, v. 68, n. 3, p. 386-392, 2011. DOI: http://dx.doi.org/10.1590/S0103-901620110003 000 17

OLIVEIRA, I. A. de; CAMPOS, M. C. C.; SOARES, M. D. R.; AQUINO, R. E. de; MARQUES JÚNIOR, J.; NASCIMENTO, E. P. do. Variabilidade espacial de atributos físicos em um cambissolo háplico, sob diferentes usos na região sul do Amazonas. Revista
Brasileira de Ciência do Solo, v. 37, n. 4, p. 1103-1112, 2013. DOI: https://doi.org/10.1590/S010006832013000400027

RODRIGUES, M. S.; CORÁ, J. E.; FERNANDES, C. Spatial relationships between soil attributes and corn yield in no-tillage system. Revista Brasileira de Ciência do Solo, v. 36, n. 2, p. 599-609, 2012. DOI: https://doi.org/10.1590/S0100-06832012000200029

SANA, R. S.; ANGHINONI, I.; BRANDÃO, Z. N.; HOLZSCHUM, M. L. Variabilidade espacial de atributos físico-químicos do solo e seus efeitos na produtividade do algodoeiro. Revista Brasileira de Engenharia Agrícola e Ambiental, v. 18, n. 10, p. 994-1002, 2014. DOI: http://dx.doi.org/10.1590/18071929/agriambi. v18n10p994-1002

SANTI, A. L.; AMADO, T. J. C.; CHERUBIN, M. R.; MARTINS, T. N.; PIRES, J. L.; DELLA FLORA, L. P.; BASSO, C. J. Análise de componentes principais de atributos químicos e físicos do solo limitantes à produtividade de grãos. Pesquisa Agropecuária Brasileira, v. 47, n. 9, p. 1346-1357, 2012. DOI: https://doi.org/10.1590/S0100-204X201200090002 0

SILVA, S. A.; LIMA, J. S. S.; SOUZA, G. S.; OLIVEIRA, R. B.; SILVA, A. F. Variabilidade espacial do fósforo e das frações granulométricas de um Latossolo Vermelho Amarelo. Revista Ciência Agronômica, v. 41, n. 1, p. 18, 2010.

SOUZA, Z. M.; CERRI, D. G. P.; MAGALHÃES, P. S. G.; SIQUEIRA, D. S. Spatial variability of soil attributes and sugarcane yield in relation to topographic location. Revista Brasileira de Engenharia Agrícola e Ambiental, v. 14, n. 12, p. 1250-1256, 2010. DOI: http:/ /dx.doi.org/10.1590/S1415-43662010001200001

SOUZA, Z. M.; MARQUES JÚNIOR, J.; PEREIRA, G. T.; BARBIERI, D. M. Variabilidade espacial da textura de um Latossolo Vermelho Amarelo eutroférrico sob cultivo de cana-deaçúcar. Engenharia Agrícola, v. 24, n. 2, p. 309-319. 2004. DOI: https:/ / doi.org/10.1590/S0100-69162004000200009

ZIMBACK, C. R. L. Análise espacial de atributos químicos de solos para fins de mapeamento da fertilidade do solo. Tese de Livre Docência. Universidade Estadual Paulista, Botucatu, 2001. 114p.

ZONTA, J. H.; MARTINEZ, M. A.; PRUSKI, F. F.; SILVA, D. D.; SANTOS, M. R. Efeito da aplicação sucessiva de precipitações pluviais com diferentes perfis na taxa de infiltração de água no solo. Revista Brasileira de Ciência do Solo, v. 36, n. 2, p. 377-388, 2012. DOI: http://dx.doi.org/10.1590/S0100-06832012000200007 